\title{
CARACTERÍSTICAS EPIDEMIOLÓGICAS, CLÎNICAS Y BACTERIOLÓGICAS DE MENINGITIS NEUMOCÓCICA EN PACIENTES PEDIÁTRICOS DE LIMA, PERÚ
}

\author{
Long Davalos ${ }^{1, a}$, Yessica Terrazas ${ }^{1,2, a}$, Ana Quintana ${ }^{1,2, a}$, Martha Egoavil ${ }^{1, a}$, Katherine Sedano ${ }^{1,2, a}$, \\ María E. Castillo ${ }^{1,3, b}$, Isabel Reyes, ${ }^{4, a}$, Eduardo Chaparro ${ }^{1,2, b}$, Wilda Silva ${ }^{5, b}$, Francisco Campos ${ }^{6, b}$, Andrés Saenz $^{7, b}$, \\ Roger Hernandez ${ }^{1,2, b}$, Olguita del Águila ${ }^{5, b}$, Daniel Guillén Pinto ${ }^{1,2, c}$, Theresa J. Ochoa ${ }^{1,8, b}$
}

\section{RESUMEN}

Objetivos. Describir las características clínicas, letalidad, susceptibilidad antibiótica y distribución de serotipos de meningitis neumocócica en pacientes pediátricos de Lima, Perú. Materiales y Métodos. Serie de casos de meningitis neumocócica en niños menores de 16 años. Los datos fueron obtenidos de dos estudios multicéntricos prospectivos, de vigilancia pasiva de enfermedad neumocócica invasiva realizados en Lima-Perú desde los años 2006 al 2008 , y del 2009 al 2011. Resultados. Reportamos 44 episodios de meningitis neumocócica; $68,2 \%$ fueron en niños menores de 2 años. La tasa de letalidad fue 32,6; y 92,9\% de los casos letales ocurrieron en niños menores de dos años $(p<0,05)$. La desnutrición estuvo asociada a los casos letales $(p<0,05)$. El $64,3 \%$ de los casos fatales murieron dentro de los 2 primeros días. El $41,9 \%$ de los cultivos con neumococo fueron resistentes a la penicilina, 23,3\% mostró resistencia intermedia a ceftriaxona (ninguno mostró resistencia completa) y $9,3 \%$ mostró resistencia a cloranfenicol. Los serotipos más frecuentes fueron $6 \mathrm{~B}, 14,19 \mathrm{~F}$ y $23 \mathrm{~F}$, los cuales constituyeron el $68,3 \%$ de todas las cepas; $84,1 \%$ de las cepas encontradas están incluidas en los serotipos de la vacuna 13 valente. Conclusiones. La meningitis neumocócica continúa siendo una enfermedad letal, especialmente en niños menores de 2 años. Dado que aproximadamente dos tercios de los casos letales fallecen en las primeras $48 \mathrm{~h}$, es crítico un diagnóstico y tratamiento oportuno, así como asegurar el cumplimiento de la inmunización con la vacuna neumocócica.

Palabras clave: Streptococcus pneumoniae; Meningitis neumocócica; Perú; Mortalidad infantil (fuente: DeCS BIREME).

\section{EPIDEMIOLOGIC, CLINICAL AND BACTERIOLOGIC CHARACTERISTICS OF PNEUMOCOCCAL MENINGITIS IN PEDIATRIC PATIENTS FROM LIMA, PERU}

\begin{abstract}
Objectives. To describe the clinical characteristics, lethality, antibiotic susceptibility, and serotype distribution of pneumococcal meningitis in pediatric patients in Lima, Peru. Materials and Methods. A case series of pneumococcal meningitis in children less than 16 years of age from two prospective, multicenter, passive surveillance studies of invasive pneumococcal diseases held in Lima-Peru from 2006 to 2008 and 2009 to 2011 . Results. We report 44 pneumococcal meningitis episodes; $68.2 \%$ of them were in children less than 2 years old. The overall case fatality rate was $32.6 \% ; 92.9 \%$ of fatal cases were in children less than 2 years of age $(p<0.05)$. Malnutrition was associated with fatal cases $(p<0.05)$. $64.3 \%$ of fatal cases died within the first two days. $41.9 \%$ of pneumococcal isolates were resistant to penicillin, $23.3 \%$ were intermediate resistant to ceftriaxone (none were highly resistant) and $9.3 \%$ were resistant to chloramphenicol. The most common serotypes were $6 \mathrm{~B}, 14,19 \mathrm{~F}$ and $23 \mathrm{~F}$, which accounted for $68.3 \%$ of all strains; $84.1 \%$ of strains were PCV13 serotypes. Conclusions. Pneumococcal meningitis continues to be a lethal disease, especially in children less than 2 years of age. Since almost two third of lethal cases lead to death within the first 48 hours, prompt diagnosis and management is critical, as well as assurance of immunization with pneumococcal vaccine.
\end{abstract}

Key words: Streptococcus pneumoniae; Meningitis, pneumococcal; Peru; Infant mortality (source: MeSH NLM).

\footnotetext{
Universidad Peruana Cayetano Heredia. Lima, Perú.

Hospital Nacional Cayetano Heredia. Lima, Perú.

Instituto Nacional de Salud del Niño. Lima, Perú.

Hospital de Emergencias Pediátricas. Lima, Perú.

Hospital Nacional Edgardo Rebagliati Martins. Lima, Perú.

Hospital Nacional Docente Madre-Niño San Bartolomé. Lima, Perú.

Hospital Daniel A. Carrión. Lima, Perú.

8 University of Texas, School of Public Health. Houston, Texas, United States of America.

a Médico cirujano, ${ }^{\mathrm{b}}$ médico infectólogo pediatra, ${ }^{\mathrm{c}}$ médico neurólogo pediatra.

Recibido: 29/02/2016 Aprobado: 06/07/2016
}

Citar como: Dávalos LF, Terrazas Y, Quintana A, Egoavil M, Sedano K, Castillo ME, et al. Características epidemiológicas, clínicas y bacteriológicas de meningitis neumocócica en pacientes pediátricos de Lima, Perú. Rev Peru Med Exp Salud Publica. 2016;33(3):425-31. doi: 10.17843/rpmesp.2016.333.2349 


\section{INTRODUCCIÓN}

El Streptococcus pneumoniae es la causa más común de meningitis bacteriana en niños mayores de 2 meses en Estados Unidos ${ }^{(1)}$. Es una causa importante de mortalidad y morbilidad infantil, que provoca una cantidad de secuelas neurológicas que incluyen parálisis cerebral, convulsiones, impedimentos motores, discapacidad intelectual, daño auditivo y pérdida de funciones cognitivas ${ }^{(2)}$.

La incidencia de meningitis neumocócica en Latino América y el Caribe fue de 4,6/100 000 en niños de 0 a 59 meses, entre los años 2000 y $2010{ }^{(3)}$. Así mismo, la incidencia de meningitis neumocócica en Estados Unidos fue de 3,66/100 000 en niños menores a 2 años y de $0,87 / 100000$ en niños entre 2 y 4 años, entre los años 2004 y $2005{ }^{(4)}$. La Organización Mundial de la Salud estimó que globalmente ocurrieron 476000 muertes en niños HIV negativo menores de 5 años a causa de infecciones por neumococo en el 2008, de los cuales 33200 fueron por meningitis neumocócica ${ }^{(5)}$. La incidencia de enfermedad neumocócica invasiva (ENI) es alta en países en desarrollo y desarrollados ${ }^{(6,7)}$. En Lima, la incidencia hospitalaria de ENI fue de 7,7/100 000 en niños menores de 60 meses, en los años 2006 al 2008, siendo meningitis la entidad más letal ${ }^{(8)}$.

En Perú, en julio de 2009, se introdujo la vacuna neumocócica conjugada 7 valente (PCV-7, en inglés: pneumococcal conjugate vaccine) en el esquema nacional de vacunación. Posteriormente, en el 2012 se introdujo la vacuna 10 valente y en el 2015 , la vacuna 13 valente (PCV13). La PCV-7 incluía los serotipos 4, 6B, 9V, 14, 18C, 19F y $23 \mathrm{~F}$; la PCV-10 incluye los serotipos de la PCV-7 más los serotipos 1, 5 y 7F; la PCV-13 incluye los de la PCV-10 más los serotipos $3,6 \mathrm{~A}$, y 19A. Las coberturas vacunales no fueron muy altas en el primer año de la introducción de la vacuna. En el 2009 se consiguió una cobertura de sólo $39,9 \%$ en Lima y de $37,9 \%$ a nivel nacional ${ }^{(9)}$.

La resistencia antibiótica y distribución de serotipos varían según país y región geográfica ${ }^{(10-12)}$. Conocer los patrones de susceptibilidad antimicrobianas locales es importante al momento de decidir el tratamiento empírico inicial; sin embargo, no existe mucha información acerca de meningitis neumocócica en el Perú. Por tal motivo, el presente estudio tiene la finalidad de describir las características clínicas, bacteriológicas y epidemiológicas de la meningitis neumocócica en hospitales de Lima, Perú.

\section{MATERIALES Y MÉTODOS}

\section{DISEÑO DEL ESTUDIO}

Este es un estudio descriptivo, reporte de serie de casos de meningitis neumocócica. Los datos fueron obtenidos del registro clínico de dos estudios prospectivos, multicéntricos de vigilancia pasiva de enfermedad neumocócica invasiva (ENI). Estos estudios fueron realizados en once hospitales públicos y cinco laboratorios privados de Lima Metropolitana, de mayo 2006 a abril $2008^{(8)}$ y de julio 2009 a junio 2011 (resultados aún no publicados) respectivamente. El primer estudio corresponde al periodo previo a la introducción de la PCV-7, mientras que el segundo, al periodo posvacuna. El diseño de investigación y población de estudio del segundo trabajo no publicado es similar al primer estudio, cuyo objetivo consistió en determinar la epidemiología de la ENI, resistencia antibiótica y distribución de serotipos en el periodo posvacuna.

De los once hospitales, ocho pertenecen al Ministerio de Salud del Perú: Instituto Nacional de Salud del Niño, Hospital de Emergencias Pediátricas, Hospital Nacional Cayetano Heredia, Hospital Nacional Docente Madre-Niño San Bartolomé, Hospital Daniel A. Carrión, Hospital María Auxiliadora, Hospital Hipólito Unanue, Hospital Arzobispo Loayza; y tres al Seguro Social de Salud (EsSalud): Hospital Edgardo Rebagliati, Hospital Guillermo Almenara y Hospital Sabogal. Los cinco laboratorios privados fueron: Blufstein, MedLab, AngloLab, Roe y Suiza Lab.

\section{DEFINICIÓN DE CASO}

Para este estudio, los casos de meningitis neumocócica fueron definidos como: aislamiento de S. pneumoniae del líquido cefalorraquídeo (LCR) o diagnóstico clínico de meningitis con aislamiento de $S$. pneumoniae en sangre ${ }^{(11)}$, en pacientes menores de 16 años admitidos a los hospitales y clínicas participantes.

\section{VARIABLES}

Se reportaron las siguientes variables: antecedentes patológicos, presencia de alguna enfermedad o condición patológica diagnosticada previa a la admisión; días de hospitalización, el número de días desde la admisión hasta el alta o fallecimiento; días de enfermedad antes del ingreso, el número de días desde el primer síntoma hasta el día de admisión; condición al alta, que incluyó las categorías de vivo, vivo con algún tipo de secuela o fallecido; y presencia de sepsis, síndrome de respuesta inflamatoria más un probable foco de infección al ingreso. Los síntomas y signos considerados para el diagnóstico clínico de meningitis fueron cefalea, fiebre, alteración del sensorio y rigidez de nuca. Estas variables fueron analizadas en dos grupos: menores de 2 años y mayor o igual a 2 años. Se utilizó este punto de corte dado que se ha observado una mayor vulnerabilidad en pacientes menores a 2 años ${ }^{(13)}$.

\section{ESTUDIOS DE LABORATORIO}

Las muestras de S. pneumoniae fueron transportados a un único laboratorio central el mismo día del 
aislamiento. La identificación de $S$. pneumoniae fue confirmada por métodos microbiológicos convencionales. La susceptibilidad antibiótica a tres antibióticos - ceftriaxona, cloranfenicol y penicilina - fueron determinados por concentración mínima inhibitoria (CMI) usando Etest ${ }^{\circledR}$ (AB Biodisk, Solna, Sweden). La susceptibilidad fue interpretada según la guía Performance Standards of the Clinical and Laboratory Standards Institute (CLSI/NCCLS) del

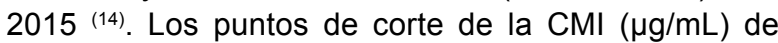
la penicilina parenteral para meningitis fueron $\leq 0,06$ susceptible $y \geq 0,12$ resistente. Para la ceftriaxona parenteral fueron $\leq 0,5$ susceptible, 1 intermedio y $\geq 2$ resistente ${ }^{(14)}$. Los serotipos fueron tipificados en el Laboratorio de Estreptococo del Centro de control y prevención de enfermedades (CDC) mediante reacción de Quellung.

Con respecto al LCR, los valores que se consideraron normales fueron: leucocitos $0-5$ células $/ \mu \mathrm{L}$, proteína $<45 \mathrm{mg} / \mathrm{dL}$ y glucosa $>60 \%$ de glucosa en sangre. Todos los procedimientos de laboratorio descritos y puntos de cortes fueron los mismos utilizados en el estudio original ${ }^{(8)}$.

\section{DETERMINACIÓN DE LA TASA DE LETALIDAD}

Se captaron todos los casos de meningitis neumocócica de las sedes y periodos previamente descritos. Se determinó el número de pacientes que fallecieron debido a meningitis neumocócica durante su estancia hospitalaria. La tasa de letalidad se estimó basada en la proporción de estos datos.

\section{ESTIMACIÓN DE LA COBERTURA ESTIMADA POR LAPCV-13}

Se calculó el porcentaje de casos debido a cepas incluidas en la PCV-13, con respecto al total de casos reportados de meningitis neumocócica en este estudio.

\section{ANÁLISIS ESTADÍSTICO}

Estudio descriptivo; los datos son presentados como número de casos y porcentajes. Se utilizó chi cuadrado con corrección de Yates para las comparaciones exploratorias. Se usó un $p<0,05$ para determinar significancia estadística.

\section{ASPECTOS ÉTICOS}

Los protocolos de los estudios originales fueron aprobados por el comité de ética de la Universidad Peruana Cayetano Heredia, y de cada uno de los hospitales participantes. Los autores de los estudios principales participaron en este trabajo, los cuales proporcionaron la base de datos.

\section{RESULTADOS}

Del 1 de mayo 2006 al 30 de abril 2008, y del 1 de julio 2009 al 30 de junio 2011, se reportaron 44 episodios de meningitis neumocócica de un total de 42 pacientes (dos pacientes tuvieron dos episodios separados de meningitis neumocócica). El rango de edad fue entre 1 mes a 13,5 años, la mediana fue 1 año, con rangos intercuartílicos de 5 meses a 3,8 años. Treinta casos de los 44 episodios reportados $(68,1 \%)$ correspondieron a pacientes menores de 2 años; 24 (54,5\%) eran hombres y $35(79,5 \%)$ fueron diagnosticados por cultivo positivo en LCR; 35 casos fueron detectados antes de la introducción de la PCV-7 al Programa Nacional de Vacunación en el Perú. De los nueve casos restantes, solo uno recibió la PCV-7 y otro la vacuna neumocócica 23 valente.

Los signos y síntomas más frecuentes fueron trastorno del sensorio, fiebre y cefalea. Alrededor de un tercio de los pacientes presentó síntomas inespecíficos como hipoactividad, tos y rinorrea. Los niños menores a 2 años fueron más propensos a tener convulsiones $(p<0,01)$. Veintinueve casos $(65,9 \%)$ presentaron sepsis y seis casos $(13,6 \%)$ presentaron shock séptico al ingreso. Se obtuvo seguimiento de 43 casos; un paciente fue transferido a otro hospital por lo que su condición al alta es desconocida. La mediana de la estancia hospitalaria fue de 15 días y 11 pacientes (26,2\%) estuvieron hospitalizados por más de 21 días; 37 niños $(84,1 \%)$ tuvieron al menos una complicación relacionada a meningitis, que incluyeron hipertensión endocraneana $(61,3 \%)$ y convulsiones $(55,8 \%)$. Diecisiete pacientes $(39,5 \%)$ fueron dados de alta sin secuelas, doce $(27,9 \%)$ tuvieron algún tipo de secuela y catorce $(32,6 \%)$ murieron (Tabla 1). Hubo un mayor número de casos fatales y secuelas en los niños menores de 2 años (Tabla 1). Las secuelas descritas fueron hipoacusia, parálisis facial, retraso del desarrollo psicomotor y epilepsia.

Se tipificó a todas las cepas y se encontró quince serotipos diferentes. Los más comunes fueron $6 \mathrm{~B}, 14$, $19 \mathrm{~F}$ y $23 \mathrm{~F}$ los cuales constituyeron el $68,3 \%$ de todas las muestras (Tabla 1). Del grupo previo a la introducción de la PCV-7 (35 casos), 30 (85,7\%) mostraron serotipos que estaban incluidos en la PCV-13. En su contraparte, del grupo de pacientes del segundo periodo (nueve casos), siete $(77,7 \%)$ estaban incluidos; sin embargo, esta diferencia no fue significativa. La cobertura estimada por la PCV-13 en los 44 casos fue del $84,1 \%$.

Cuarenta $(90,9 \%)$ muestras de LCR fueron estudiadas. La mediana del número de leucocitos en LCR fue 418 células $/ \mathrm{mm}^{3}$, veintiuno $(52,5 \%)$ fueron menores a 500 células $/ \mathrm{mm}^{3}$ y aproximadamente un quinto de los pacientes tuvieron menos de 100 células $/ \mathrm{mm}^{3} ; 62,1 \%$ tuvieron predominancia polimorfonuclear. Con respecto a 
Tabla 1. Características clínicas y distribución de serotipos según edad en pacientes menores de 16 años con meningitis neumocócica. Lima, Perú

\begin{tabular}{|c|c|c|c|c|}
\hline & $<2$ años & $\geq 2$ años & Total & Valor $n$ \\
\hline & $n=30(\%)$ & $n=14(\%)$ & $n=44(\%)$ & valor $p$ \\
\hline \multicolumn{5}{|l|}{ Síntomas y signos iniciales } \\
\hline Transtorno del sensorio & $30 / 30(100,0)$ & $12 / 14(85,7)$ & $42 / 44(95,5)$ & 0,17 \\
\hline Fiebre & $28 / 30(93,3)$ & $13 / 14(92,9)$ & $41 / 44(93,2)$ & 1 \\
\hline Cefalea & NA & $11 / 13(84,6)$ & $11 / 13(84,6)$ & NA \\
\hline Rigidez de nuca & $3 / 5(60,0)$ & $11 / 14(78,6)$ & $14 / 19(73,7)$ & $0,57^{*}$ \\
\hline Vómitos & $19 / 30(63,3)$ & $13 / 14(92,9)$ & $32 / 44(72,7)$ & 0,09 \\
\hline Signos meningeos & $2 / 5(40,0)$ & $8 / 14(57,1)$ & $10 / 19(52,6)$ & $0,63^{*}$ \\
\hline Convulsiones & $17 / 29(58,6)$ & $1 / 14(7,1)$ & $18 / 43(41,9)$ & $<0,01^{*}$ \\
\hline Tos y rinorrea & $11 / 30(37,5)$ & $6 / 14(42,9)$ & $17 / 44(38,6)$ & 0,95 \\
\hline Hipoactividad & $9 / 24(37,5)$ & $2 / 11(18,2)$ & $11 / 35(31,4)$ & $0,44^{*}$ \\
\hline Irritabilidad & $7 / 24(29,2)$ & $3 / 11(27,3)$ & $10 / 35(28,6)$ & $1^{*}$ \\
\hline Diarrea & $8 / 30(26,7)$ & $1 / 14(7,1)$ & $9 / 44(20,5)$ & $0,23^{*}$ \\
\hline \multicolumn{5}{|l|}{ Días de enfermedad antes del ingreso } \\
\hline Mediana (rango) & $3(0,5-14)$ & $3(0,6-10)$ & $3(0,5-14)$ & \\
\hline$<1$ día & $4 / 29(13,8)$ & $4 / 14(28,6)$ & $8 / 43(18,6)$ & $0,40^{*}$ \\
\hline 1-3 días & $12 / 29(41,4)$ & $8 / 14(57,1)$ & $20 / 43(46,5)$ & 0,51 \\
\hline$>3$ días & $13 / 29(44,8)$ & $4 / 14(28,6)$ & $17 / 43(39,5)$ & $0,34^{*}$ \\
\hline \multicolumn{5}{|l|}{ Días de hospitalización } \\
\hline Mediana (rango) & $13(0.3-44)$ & $16(0.4-35)$ & $15(0,3-44)$ & \\
\hline >21 días & $8 / 29(27,6)$ & $3 / 13(23,1)$ & $11 / 42(26,2)$ & $1^{*}$ \\
\hline \multicolumn{5}{|l|}{ Condición al alta ${ }^{\dagger}$} \\
\hline Vivo sin secuelas & $7 / 29(24,1)$ & $10 / 14(71,4)$ & $17 / 43(39,5)$ & \\
\hline Vivo con secuelas ${ }^{\ddagger}$ & $9 / 29(31,0)$ & $3 / 14(21,4)$ & $12 / 43(27,9)$ & $<0,01^{\S}$ \\
\hline Fallecidos & $13 / 29(44,8)$ & $1 / 14(7,1)$ & $14 / 43(32,6)$ & \\
\hline \multicolumn{5}{|l|}{ Serotipos } \\
\hline $6 \mathrm{~B}$ & $8 / 30(26,7)$ & $1 / 14(7,1)$ & $9 / 44(20,5)$ & $0,23^{*}$ \\
\hline 14 & $7 / 30(23,3)$ & $2 / 14(14,3)$ & $9 / 44(20,5)$ & $0,70^{*}$ \\
\hline $19 \mathrm{~F}$ & $4 / 30(13,3)$ & $4 / 14(28,6)$ & $8 / 44(18,2)$ & $0,24^{*}$ \\
\hline $23 \mathrm{~F}$ & $3 / 30(10,0)$ & $1 / 14(7,1)$ & $4 / 44(9,1)$ & $1^{*}$ \\
\hline $18 \mathrm{C}$ & $1 / 30(3,3)$ & $1 / 14(7,1)$ & $2 / 44(4,5)$ & $0,54^{*}$ \\
\hline $19 \mathrm{~A}$ & $1 / 30(3,3)$ & $0 / 14(0)$ & $1 / 44(2,3)$ & $1^{*}$ \\
\hline 1 & $0 / 30(0)$ & $1 / 14(7,1)$ & $1 / 44(2,3)$ & $0,32^{*}$ \\
\hline 5 & $1 / 30(3,3)$ & $0 / 14(0)$ & $1 / 44(2,3)$ & $1^{*}$ \\
\hline $9 \mathrm{~V}$ & $1 / 30(3,3)$ & $0 / 14(0)$ & $1 / 44(2,3)$ & $1^{*}$ \\
\hline Otros no incluidos en PCV-13" & $4 / 30(13,3)$ & $3 / 14(21,4)$ & $7 / 44(15,9)$ & $0,66^{*}$ \\
\hline
\end{tabular}

* Valor $p$ calculado con prueba exacta de Fisher.

† Un paciente fue transferido a otro hospital.

‡ Las secuelas incluyeron: hipoacusia, parálisis facial, retraso del desarrollo psicomotor, epilepsia.

$\S$ Valor p calculado con Chi cuadrado para tendencias.

" Los serotipos no incluidos en la PCV-13 fueron 12F, 13, 15A, 16F, 34, 38.

NA: No aplica

la concentración proteica, la mediana fue $245 \mathrm{mg} / \mathrm{dL}$ y $100 \%$ fueron mayores a $50 \mathrm{mg} / \mathrm{dL}$. El $72,5 \%$ presentó una glucosa menor a $40 \mathrm{mg} / \mathrm{dL}$ y $81,3 \%$ una relación LCR/glucosa sérica menor a 0,5. En la tinción Gram se observó diplococos Gram positivos en el $84,2 \%$ de los casos (Tabla 2 ).

La tasa de letalidad fue $32,6 \%$; de los catorce fallecidos, el $92,9 \%$ ocurrió en menores de 2 años $(p=0,03)$, nueve $(64,3 \%)$ fallecieron dentro de los dos primeros días. La desnutrición estuvo asociada a mortalidad $(p=0,03)$; no hubieron otras diferencias significativas entre los casos fatales y no fatales (Tabla 3 ). A veinticinco pacientes $(56,8 \%)$ se les hizo tomografía cerebral, de los cuales dieciocho $(72,0 \%)$ mostraron resultados anormales. Los hallazgos más frecuentes fueron edema cerebral $(24,0 \%)$, colección subdural $(20,0 \%)$, aumento del espacio subaracnoideo $(12,0 \%)$ y ventriculomegalia/hidrocefalia $(8,0 \%)$.

El $40,9 \%$ de los cultivos con neumococo mostró resistencia a la penicilina y $22,7 \%$ mostró resistencia 
Tabla 2. Características en LCR según edad en pacientes menores de 16 años con meningitis neumocócica*. Lima, Perú

\begin{tabular}{|c|c|c|c|c|}
\hline \multirow{2}{*}{ Valores en LCR } & $<2$ años & $\geq 2$ años & Total & \multirow{2}{*}{ Valor $p$} \\
\hline & $n=26(\%)$ & $n=14(\%)$ & $n=40(\%)$ & \\
\hline \multicolumn{5}{|l|}{ Leucocitos (células $/ \mathrm{mm}^{3}$ ) } \\
\hline Mediana (rango) & $489(2-13800)$ & $375(53-18000)$ & $418(2-18000)$ & \\
\hline$<100$ & $6 / 26(23,1)$ & $1 / 14(7,1)$ & $7 / 40(17,5)$ & $0,38+$ \\
\hline$<500$ & $13 / 26(50,0)$ & $8 / 14(57,1)$ & $21 / 40(52,5)$ & 0,92 \\
\hline$>50 \%$ PMN & $11 / 19(57,9)$ & $7 / 10(70,0)$ & $18 / 29(62,1)$ & 0,81 \\
\hline \multicolumn{5}{|l|}{ Proteínas (mg/dL) } \\
\hline Mediana (rango) & $269(93-1155)$ & $147(106-480)$ & $245(93-1155)$ & \\
\hline$>150$ & $21 / 26(80,8)$ & $6 / 13(46,2)$ & $27 / 39(69,2)$ & 0,065 \\
\hline \multicolumn{5}{|l|}{ Glucosa (mg/dL) } \\
\hline Mediana (rango) & $9(1-90)$ & $15(0-74)$ & $10(0-90)$ & \\
\hline$<40$ & $20 / 26(76,9)$ & $9 / 14(64,3)$ & $29 / 40(72,5)$ & 0,62 \\
\hline LCR/Suero $<0.5$ & $17 / 21(81,0)$ & $9 / 11(81,8)$ & $26 / 32(81,3)$ & 1 \\
\hline Tinción Gram positiva & $23 / 26(88,5)$ & $9 / 12(75,0)$ & $32 / 38(84,2)$ & 0,56 \\
\hline
\end{tabular}

*Se obtuvo muestra de líquido cefalorraquideo (LCR) en 40 casos. Los cuatro casos restantes fueron diagnosticados mediante criterio clínico y cultivo en sangre positivo.

† Valor $p$ calculado con prueba exacta de Fisher.

intermedia a ceftriaxona; ninguno mostró resistencia completa a ceftriaxona. Una alta proporción de los cultivos fue sensible a cloranfenicol $(90,9 \%)$. El serotipo 14 estuvo asociado a una mayor resistencia a la penicilina $(p=0,032)$. No hubo asociación entre resistencia antibiótica y edad o letalidad. El antibiótico más usado como terapia inicial fue ceftriaxona $(68,1 \%)$ y ceftriaxona más vancomicina $(15,9 \%)$. Otros antibióticos usados fueron ampicilina $(4,5 \%)$, ceftriaxona más amikacina $(4,5 \%)$ y ceftriaxona más meropenem $(2,3 \%)$.

Tabla 3. Características de pacientes menores de 16 años con meningitis neumocócica según letalidad*. Lima, Perú

\begin{tabular}{|c|c|c|c|}
\hline & Fallecidos & Vivos & \\
\hline & $\mathrm{n}=14(\%)$ & $\mathrm{n}=29(\%)$ & Valor $p$ \\
\hline \multicolumn{4}{|l|}{ Edad } \\
\hline$<2$ años & $13 / 14(92,9)$ & $16 / 29(55,2)$ & 0,03 \\
\hline \multicolumn{4}{|l|}{ Tiempo de enfermedad } \\
\hline$<1$ día & $2 / 13(15,4)$ & $3 / 29(10,3)$ & $0,64^{\dagger}$ \\
\hline >3 días & $5 / 13(38,5)$ & $12 / 29(41,4)$ & 1 \\
\hline \multicolumn{4}{|l|}{ Antecedentes patológicos } \\
\hline Desnutrición ${ }^{\ddagger}$ & $5 / 13(38,5)$ & $2 / 29(6,9)$ & 0,03 \\
\hline Cardiopatía & $1 / 13(7,7)$ & $2 / 29(6,9)$ & $1^{\dagger}$ \\
\hline Enfermedad bronco-obstructiva & $2 / 13(15,4)$ & $5 / 29(17,2)$ & $1^{\dagger}$ \\
\hline \multicolumn{4}{|l|}{ Síntomas y signos } \\
\hline Vómitos & $7 / 14(50,0)$ & $24 / 29(82,8)$ & 0,06 \\
\hline Fiebre & $13 / 14(92,9)$ & $27 / 29(93,1)$ & 1 \\
\hline Convulsiones & $7 / 13(53,8)$ & $10 / 29(34,5)$ & 0,39 \\
\hline Trastorno del sensorio & $14 / 14(100,0)$ & $27 / 29(93,1)$ & 0,81 \\
\hline \multicolumn{4}{|l|}{ Características del LCR } \\
\hline Leucocitos $<100$ células/ $\mu \mathrm{L}$ & $3 / 11(27,3)$ & $4 / 28(14,3)$ & $0,38^{\dagger}$ \\
\hline Proteínas $>50 \mathrm{mg} / \mathrm{dL}$ & $11 / 11(100,0)$ & $27 / 27(100,0)$ & 1 \\
\hline Glucosa LCR/sangre $<40 \%$ & $6 / 11(54,5)$ & $22 / 28(78,6)$ & 0,26 \\
\hline \multicolumn{4}{|l|}{ Resistencia antibiótica } \\
\hline Resistente a penicilina & $4 / 14(28,6)$ & $14 / 29(48,3)$ & $0,32^{\dagger}$ \\
\hline Resistente a ceftriaxona ${ }^{\S}$ & $4 / 14(28,6)$ & $6 / 29(21,7)$ & $0,70^{\dagger}$ \\
\hline Resistente a cloranfenicol & $3 / 14(21,4)$ & $1 / 29(3,4)$ & $0,09^{\dagger}$ \\
\hline
\end{tabular}

* Se obtuvo la condición al alta en 43 pacientes. El paciente restante fue transferido a otro hospital.

† Valor $p$ calculado con prueba exacta de Fisher.

¥ Desnutrición aguda fue definida como peso para talla por debajo de -2 puntuaciones Z y desnutrición crónica como talla para edad por debajo de -2 puntuaciones Z.

$\S$ Resistencia intermedia a ceftriaxona. 


\section{DISCUSIÓN}

La meningitis neumocócica en la infancia es una enfermedad mortal que tiene las tasas de mortalidad más altas entre los pacientes con enfermedad neumocócica invasiva (7,15). La tasa de letalidad en América Latina y el Caribe es de $33 \%{ }^{(3)}$, la cual es similar a la letalidad encontrada en este estudio. Estas cifras son alarmantes, especialmente si más del $50 \%$ de los casos fatales muere durante las dos primeras horas del ingreso. Por ende, es crucial detectar y tratar la meningitis neumocócica oportunamente para disminuir las tasas de mortalidad.

Los médicos deben estar al tanto del espectro clínico de esta enfermedad para evitar errores en el diagnóstico. Los signos más frecuentes son fiebre y trastorno del sensorio. Sin embargo, un tercio de nuestros casos presentó síntomas como vómitos, hipoactividad e irritabilidad, reflejando que esta entidad puede tener presentaciones no específicas siendo difícil su diagnóstico ${ }^{(16,17)}$. Los niños menores a 2 años son el grupo más vulnerable ${ }^{(13)}$ pues sus síntomas neurológicos son poco específicos y los signos meníngeos no siempre están presentes. Estos hechos conllevan a retrasos en el diagnóstico y, por ende, mayor mortalidad y alta frecuencia de secuelas. De manera interesante, este grupo fue más propenso a desarrollar convulsiones (18), constituyendo el $58,6 \%$ en nuestra serie, lo cual es mayor a las cifras registradas en otros países ${ }^{(13,16)}$. Por esta razón, las convulsiones deben ser un signo de alarma y aumentar la sospecha de meningitis neumocócica cada vez que se presenten.

El análisis de LCR es una herramienta importante en el diagnóstico de meningitis neumocócica; sin embargo, debemos ser cuidadosos al interpretar los resultados. A diferencia de otros estudios (13,19), la mediana de leucocitos en LCR fue 418 células $/ \mathrm{mm}^{3}$ y solo el $62,1 \%$ de muestras presentó predominancia polimorfonuclear. Ello indica que la ausencia de pleocitosis y la predominancia linfomononuclear no excluyen meningitis bacteriana. A pesar que una pobre respuesta leucocitaria en LCR está relacionada a mal pronóstico ${ }^{(20)}$, nuestros resultados no encontraron asociación alguna entre letalidad y número de leucocitos en LCR. La sensibilidad de los estudios en LCR no es $100 \%{ }^{(21)}$ y en el 10 al $28 \%$ de casos de meningitis, los estudios iniciales en LCR pueden mostrar valores normales, especialmente si el LCR es obtenido al inicio de la enfermedad (dentro de las primeras 24-36 h) ${ }^{(21)}$. Estos hechos enfatizan la necesidad de comenzar terapia antibiótica siempre que se tenga la sospecha clínica de meningitis bacteriana, incluso si el LCR inicial no revela resultados compatibles con meningitis bacteriana. Cabe mencionar que la tinción Gram fue positiva en el $84,2 \%$ de pacientes; esta prueba es rápida, económica y ha mostrado una buena sensibilidad en los casos de meningitis neumocócica (22), siendo una herramienta diagnóstica muy valiosa.
Durante los últimos 20 años, el aumento de la resistencia antibiótica se ha convertido en un serio problema por la falla de tratamiento y el aumento de costos de cuidados médicos ${ }^{(10)}$. En muchos países de Sudamérica como Argentina y Colombia se han reportado altas tasas de resistencia a penicilina ${ }^{(11,23)}$, así como a resistencia intermedia a ceftriaxona ${ }^{(23)}$. A pesar que nuestros resultados no mostraron resistencia completa a ceftriaxona, es de resaltar el alto porcentaje de resistencia intermedia, sobre todo cuando la ceftriaxona es el tratamiento empírico más común en nuestro medio.

La tipificación de serotipos es esencial para una vigilancia epidemiológica precisa en el contexto de la introducción de la vacuna. La cobertura estimada por la vacuna conjugada 13 valente (PCV-13) en nuestro estudio fue del $84,1 \%$, con una pequeña reducción en el grupo posvacuna. Una distribución similar de serotipos se ha observado en América Latina, con una gran cobertura por la PCV-13 antes y después de la implementación de la vacuna ${ }^{(8,11)}$. Con mayores coberturas en el programa nacional de vacunación con la PCV-13, habrá una mayor disminución en la incidencia de esta entidad, y por ende, un gran impacto en la reducción de la mortalidad peruana infantil.

Nuestro estudio posee algunas limitaciones. Primero, los datos fueron obtenidos de un estudio de vigilancia pasiva, de pacientes hospitalizados que ingresan con cuadros clínicos más severos, lo cual puede conllevar a una sobre estimación de la tasa de letalidad. Segundo, hubo datos que no se pudieron recuperar de las fuentes originales. Tercero, debido al pequeño tamaño muestral y método de estudio, el análisis de correlación fue difícil de obtener, así como comparar la resistencia antibiótica, serotipos y terapéutica entre los períodos pre y posvacunación. Cuarto, debido al diseño del estudio, es difícil extrapolar los datos a la población en general (validez externa).

En conclusión, nuestro estudio muestra una alta tasa de letalidad de meningitis neumocócica en la población estudiada, siendo los menores de 2 años los más afectados. Si bien la evaluación del LCR es un importante método diagnóstico, la sospecha clínica debe ser la herramienta más fidedigna que nos ayude a decidir si un paciente debe iniciar tratamiento o no. Este enfoque, en conjunto con una apropiada cobertura de inmunización, conllevará a una considerable reducción de nuestra letalidad de meningitis neumocócica local. Estudios posteriores deben enfocarse en una mejor descripción de la resistencia y cambios de serotipos en la era posvacuna.

Agradecimientos: los autores agradecen a los directores y al equipo de los laboratorios de microbiología, y a los pediatras de los hospitales y clínicas participantes en el estudio.

Contribución de los autores: $L D, Y T, A Q, M E, K S, M C, E C$, WS, FC, AS y TJO participaron en la concepción y diseño del artículo. ME, MC, EC, WS, FC, AS, RH y OA aportaron con pacientes para el estudio. LD, YT, AQ, ME, KS, MC, IR, EC, WS, FC, AS y TJO participaron en la recolección/obtención de 
resultados. $\mathrm{LD}, \mathrm{YT}, \mathrm{AQ}, \mathrm{KS}$ y $\mathrm{TJO}$ analizaron e interpretaron datos. $L D, Y T, A Q$ y TJO redactaron el artículo. TJO obtuvo financiamiento. DG asesoró técnicamente en el estudio. Todos los autores revisaron en forma crítica las versiones del manuscrito y aprobaron su versión final.

Fuentes de financiamiento: beca de investigación otorgada por Laboratorios Wyeth/Pfizer (Lima, Perú), Fondo de Apoyo a la Investigación de la Facultad de Medicina Alberto Hurtado, Universidad Peruana Cayetano Heredia y Fondo de Apoyo a la Investigación del Instituto Nacional de Salud del Niño.

Conflictos de interés: TJO, MC, WS, RH y EC han sido consultantes de Laboratorios Wyeth/Pfizer. TJO ha recibido una beca de investigación de Wyeth/Pfizer.

\section{REFERENCIAS BIBLIOGRÁFICAS}

1. Thigpen MC, Whitney CG, Messonnier NE, Zell ER, Lynfield R, Hadler JL, et al. Emerging infections programs network. Bacterial meningitis in the United States, 1998-2007. N Engl J Med. 2011;364 (21):2016-2025. doi: 10.1056/ NEJMoa1005384

2. Christie D, Viner RM, Knox K, Coen PG, Wang $\mathrm{H}$, El Bashir $\mathrm{H}$, et al. Long-term outcomes of pneumococcal meningitis in childhood and adolescence. Eur J Pediatr. 2011;170(8):997. doi: 10.1007/ s00431-010-1390-5

3. Ciaponni A, Elorriaga N, Rojas JI, Romano M, Martí SG, Bardach A, et al. Epidemiology of pediatric pneumococcal meningitis and bacteremia in Latin America and the Caribbean: a systematic review and meta-analysis. Pediatr Infect Dis J. 2014;33(9):971-8. doi: 10.1097/ INF.0000000000000363

4. Hsu HE, Shutt KA, Moore MR, Beall BW, Bennett NM, Craig AS, et al. Effect of pneumococcal conjugate vaccine on pneumococcal meningitis. N Engl J Med. 2009;360(3):244-56. doi: 10.1056/NEJMoa0800836

5. World Health Organization. Estimated Hib and pneumococcal deaths for children under 5 years of age, 2008 [Internet]. Geneva: WHO; 2008 [updated 2013 Dec 1; citado el 12 de diciembre de 2015]. Disponible en: http://www.who.int/immunization/ monitoring_surveillance/burden/ estimates/Pneumo_hib/en/index. html

6. Campbell JD, Kotloff KL, Sow SO, Tapia M, Keita MM, Keita T, et al. Invasive pneumococcal infections among hospitalized children in Bamako, Mali. Pediatr Infect Dis J. 2004;23(7):642-9

7. Chong CY, Koh-Cheng T, Yee-Hui M, Nancy TW. Invasive pneumococcal disease in Singapore children. Vaccine. 2008;26(27-28):3427-31. doi: 10.1016/j.vaccine.2008.04.035
8. Ochoa TJ, Egoavil M, Castillo ME, Reyes I, Chaparro E, Silva W, et al. Invasive pneumococcal diseases among hospitalized children in Lima, Peru. Rev Panam Salud Publica. 2010;28(2):121-7

9. Perú, Ministerio de Salud. Tercer Informe. Intervención pública evaluada: servicio de vacunación [Internet]. Lima: MINSA; 2011 [citado el 15 de Agosto del 2016]. Disponible en: https://www. mef.gob.pe/contenidos/presu_publ/ ppr/eval_indep/2010_informe_final_ VACUNACIONES.pdf

10. Valenzuela MT, de Quadros C. Antibiotic resistance in Latin America: a cause for alarm. Vaccine. 2009;27 Suppl 3:C25-8. doi: 10.1016/j.vaccine.2009.06.005

11. Camargos P, Fischer G, Mocelin H, Dias C, Ruvinsky R. Penicillin resistance and serotyping of Streptococcus pneumoniae in Latin America. Paediatr Respir Rev. 2006;7(3):209-14.

12. MercadoEH, Egoavil M, HornaSG, Torres $\mathrm{N}$, Velásquez R, Castillo ME, et al. Serotipos de neumococo en niños portadores antes de la vacunación antineumocócica en el Perú. Rev Peru Med Exp Salud Publica. 2012;29(1):53-60

13. Pagliano P, Fusco U, Attanasio V, Rossi M, Pantosti A, Conte M, et al. Pneumococcal meningitis in childhood: a longitudinal prospective study. FEMS Immunol Med Microbiol. 2007;51(3):488-95.

14. Clinical and Laboratory Standards Institute (CLSI). Performance standards for antimicrobial susceptibility testing; twenty-fifth informational supplement. CLSI document M100-S25. Wayne PA: CLSI; 2015.

15. Abate HJ, Falaschi A, Balbi L, Garcia B. Nineteen-years of pneumococcal invasive disease surveillance in a children's hospital in Mendoza, Argentina. Arch Argent Pediatr. 2014;112(4):352-7.

16. Klobassa DS, Zoehrer B, Paulke-Korinek M, Gruber-Sedlmayr U, Pfurtscheller $\mathrm{K}$, Strenger V, et al. The burden of pneumococcal meningitis in Austrian children between 2001 and 2008. Eur
J Pediatr. 2014;173(7):871-8. doi: 10.1007/s00431-013-2260-8.

17. Mencía Bartolomé S, Casado Flores J, Marín Barba C, González-Vicent M, Ruiz López MJ. Meningitis neumocócica en la infancia. Revisión de 28 casos. An Esp Pediatr. 2000;53(2):94-9.

18. Lovera D, Arbo A. Risk factors for mortality in Paraguayan children with pneumococcal bacterial meningitis. Trop Med Int Health. 2005;10(12):1235-41.

19. Berberian G, Perez MG, Epelbaum C, Ceinos Mdel C, Lopardo H, Rosanova MT. Meningitis neumocócica: experiencia de 12 años en un hospital pediátrico, previa a la inmunización universal con vacuna conjugada. Arch Argent Pediatr. 2014;112(4):332-6. doi: 10.1590/S0325-00752014000400007.

20. Roine I, Pelkonen T, Bernardino L, Leite M, Kataja M, Pitkäranta A, et al. Factors affecting time to death from start of treatment among children succumbing to bacterial meningitis. Pediatr Infect Dis J. 2014;33(8):789-92. doi: 10.1097/ INF.0000000000000350.

21. Ray B, Rylance G. QUESTION 1. Normal cerebrospinal fluid: does it exclude meningitis? Arch Dis Child. 2009;94(12):988-91. doi: 10.1136/ adc. 2009.163766

22. King BA, Richmond P. Pneumococcal meningitis: clinical course and resource use in Western Australian children. J Paediatr Child Health. 2004;40(11):606-10.

23. Grenon SL, Salvi Grabulosa MC, Regueira MM, Fossati MS, von Specht MH. Meningitis neumocócica en niños menores de 15 años. Dieciséis años de vigilancia epidemiológica en Misiones, Argentina. Rev Argent Microbiol. 2014;46(1):14-23. doi: 10.1016/ S0325-7541(14)70042-2

Correspondencia: Theresa J. Ochoa Instituto de Medicina Tropical "Alexander von Humboldt" Departamento de Pediatria Universidad Peruana Cayetano Heredia

Dirección: Av. Honorio Delgado 430, San Martin de Porres, Lima 31, Perú

Teléfono: 51-1-319-0000 ext 2715

E-mail:Theresa.J.Ochoa@uth.tmc.edu 